\title{
Preparation and Characterization of a PVC/TTF-TCNQ Composite Electrode. Applications in a Flow Injection System
}

\author{
Manuel Cano, Beatriz Palenzuela and Rafael Rodríguez-Amaro* \\ Departamento de Química Física y Termodinámica Aplicada, Universidad de Córdoba, \\ Campus de Rabanales, Edificio C-3, 14071 Córdoba, España
}

\begin{abstract}
It was prepared a new polyvinyl chloride (PVC)/tetrathiafulvalenetetracyanoquinodimethane (TTF-TCNQ) composite electrode which showed good physical and electrochemical characteristics. Different PVC/TTF-TCNQ-graphite proportions were tested and the relation $1 / 10(\mathrm{p} / \mathrm{p})$ PVC/TTF-TCNQ provided the best results in terms of sensitivity, coefficients of variation and mechanical resistance. This electrode has interesting applications as a working electrode. So, employment is remarkable in a flow injection system and had been satisfactory checked to detect ascorbic acid. Also, the electrode exhibits selective voltammetric behaviour in aqueous solution that affords its use as an ion detector.

The proposed electrode showed good kinetics property with a low background current and a relatively reproducible signal. Moreover, the electrode can be prepared in a simple way and its surface easily recovered.
\end{abstract}

Keywords: TTF-TCNQ; composite electrode; PVC; ascorbic acid; flow injection.

\section{Introducción}

Las sales orgánicas conductoras (SOC) tales como tetratiofulvaleno tetracianoquinodimetano (TTF-TCNQ) poseen interesantes propiedades electroquímicas, puestas de manifiesto en su empleo como materiales electródicos, bien permitiendo una transferencia electrónica directa con compuestos electroactivos, o bien actuando como mediadores en la construcción de nuevos electrodos. Tales electrodos pueden construirse a partir de varios sustratos incluyendo $(a)$ una SOC en forma de pastillas compactas $[1,2]$ o monocristales macroscópicos [3]; (b) mezclas de una SOC con pasta de carbón [4, 5]; y (c) pasta polimérica mezclada con TTF-TCNQ y PVC en THF [6-9]. Sin embargo, los electrodos resultantes carecen tanto de estabilidad mecánica

\footnotetext{
* Corresponding author. E-mail address: qf1roamr@uco.es
} 
como de la reproducibilidad requerida para ser usados como detectores en sistemas de flujo, técnica que ha ganado una creciente importancia debido al necesario aumento de la automatización de los procesos analíticos y su control [10].

Aunque algunos electrodos compósitos de TTF-TCNQ desarrollados recientemente pueden usarse con éxito en sistemas de inyección en flujo, por ejemplo, los obtenidos mediante introducción de una sal orgánica conductora a través de una película no conductora de polipirrol para formar una estructura tridimensional [11], o mezclando TTF-TCNQ con resina epoxy, polvo de grafito y glucosa oxidasa [12], sin embargo, estos electrodos son técnicamente difíciles de preparar, y útiles sólo para aplicaciones determinadas.

El principal objetivo de este trabajo ha sido la preparación de un nuevo electrodo compósito, susceptible de ser empleado como electrodo de trabajo para la detección amperométrica en análisis de inyección en flujo, tomando como base las buenas propiedades de estabilidad de los conocidos electrodos de PVC/grafito $[13,14]$, y de la alta conductividad eléctrica de las sales orgánicas conductoras. Este artículo presenta el procedimiento de preparación y el estudio electroanalítico del electrodo compósito, donde la sal TTF-TCNQ ha sido incorporada en el interior de un compósito de PVC/grafito. La sustitución como fase conductora del grafito por la SOC aumenta la flexibilidad, selectividad y sensibilidad del electrodo compósito. Su aplicabilidad, como electrodo de trabajo, ha sido probada en la detección del ácido ascórbico mediante un sistema de flujo, obteniéndose mejoras en la respuesta voltamperométrica $\mathrm{y}$ amperométrica frente a un electrodo convencional de PVC/grafito. Este tipo de electrodo compósito también ha sido ya aplicado satisfactoriamente como sensor voltamétrico de iones [15].

\section{Parte Experimental \\ Reactivos y disoluciones}

El electrodo compósito PVC/TTF-TCNQ se construyó a partir de polvo de grafito de 1-2 micras (Aldrich), polivinil cloruro (PVC) de bajo peso molecular (Fluka de calidad puro), N,N-dimetilformamida (DMF, Merck) y la sal tetratiofulvaleno 7,7,8,8-tetracianoquinodimetano (TTF-TCNQ, Fluka de grado puro).

Todos los demás reactivos eran de calidad analítica, y las disoluciones se prepararon con agua ultrapura de un sistema Milli-Q (Millipore).

\section{Aparatos y electrodos}

Las intensidades de corriente fueron medidas con un potenciostato EG\&G PAR Modelo 273, con corrección automática de la compensación $\mathrm{iR}_{\mathrm{u}}$. Los electrodos de referencia y auxiliar usados en los ensayos en célula eran $\mathrm{Ag} / \mathrm{AgCl} 3 \mathrm{M} \mathrm{KCl}$ (Metrohm 6.0733.100) y platino (Metrohm 6.0302.100), respectivamente. El montaje instrumental era controlado informáticamente mediante el software de investigación electroquímica M270. 
El sistema de flujo consistía en una bomba peristáltica Minipuls 3 (Gilson), una válvula de seis vías (Omnifit), tubos de PTFE $0.5 \mathrm{~mm}$ de diámetro interno (Omnifit) y una celda de flujo Metrohm modelo 6.5303.020, que integraba los tres electrodos auxiliar, referencia y de trabajo. Una prensa Hidráulica (PerkinElmer) se usaba para preparar pequeñas pastillas cilíndricas de $2.5 \mathrm{~mm}$ de espesor y $4.1 \mathrm{~mm}$ de diámetro.

Todos los experimentos fueron desarrollados en condiciones ambientales ( $\mathrm{ca} .25$ $\left.{ }^{\circ} \mathrm{C}\right)$.

\section{Preparación del electrodo compósito}

Inicialmente, el electrodo compósito fue preparado a partir de pastillas obtenidas mediante mezcla directa de PVC/grafito y la sal TTF-TCNQ. Sin embargo, este procedimiento resultaba ineficaz, puesto que los electrodos obtenidos carecían de homogeneidad y resistencia mecánica, lo que provocaba una alta variabilidad en la respuesta voltamperométrica. Por este motivo, las pastillas de PVC/TTFTCNQ-grafito se prepararon siguiendo un procedimiento alternativo, similar al descrito previamente por Luque et al. [16]. En primer lugar, el polvo de polivinil cloruro era disuelto en $7.5 \mathrm{ml}$ de $N, N$-dimetilformamida, y posteriormente, se añadían $250 \mathrm{mg}$ de una proporción determinada de TTF-TCNQ/grafito para obtener una mezcla homogénea. La suspensión resultante se separaba y se secaba perfectamente a temperaturas inferiores a $60{ }^{\circ} \mathrm{C}$. El sólido así obtenido se molía en un mortero hasta formar un polvo compósito, y una cantidad de $50 \mathrm{mg}$ de este polvo se prensaba para obtener una pastilla cilíndrica. La pastilla resultante se introducía en el interior de un tubo de Teflon, en el que se introducía un tornillo de cobre para asegurar el contacto eléctrico. La fracción de área efectiva se determinó voltamperométricamente con ferrocianuro potásico en $\mathrm{KBr} 1 \mathrm{M}$, resultando ser prácticamente 1 en todos los casos.

Todas las medidas electroanalíticas eran precedidas de un proceso de limpieza de la superficie del electrodo, que consistía en (a) lijado con papel abrasivo P-1200 para obtener una superficie lisa; (b) pulido con papel satinado hasta obtener una superficie homogénea; y (c) enjuague con agua.

\section{Resultados y discusión}

El comportamiento electroanalítico del electrodo compósito se caracterizó usando ferrocianuro potásico como compuesto modelo, y la voltamperometría cíclica como técnica de medida. En primer lugar, se determinó la composición óptima del electrodo usando cantidades crecientes de SOC. Posteriormente, se modificó la proporción de PVC con objeto de asegurar el buen comportamiento analítico y mecánico.

\section{Comportamiento electroquímico del electrodo de PVC/grafito/TTF-TCNQ}

Influencia de la composición del electrodo en la respuesta analítica

La Fig. 1(d) muestra el voltagrama típico obtenido con el electrodo de $\mathrm{PVC} /$ grafito para una concentración $5 \cdot 10^{-2} \mathrm{M}$ de ferrocianuro potásico en $\mathrm{KBr} 1$ $\mathrm{M}$ como electrolito soporte, usando una velocidad de barrido de $0.01 \mathrm{~V} \mathrm{~s}^{-1}$. El 
grafito fue gradualmente reemplazado en el electrodo compósito por una cantidad equivalente de la sal TTF-TCNQ. En este sentido, se prepararon varios electrodos compósitos con una relación variable de grafito/TTF-TCNQ, manteniendo una proporción constante de PVC, con objeto de examinar el efecto de la SOC en el comportamiento electroquímico y mecánico del electrodo.

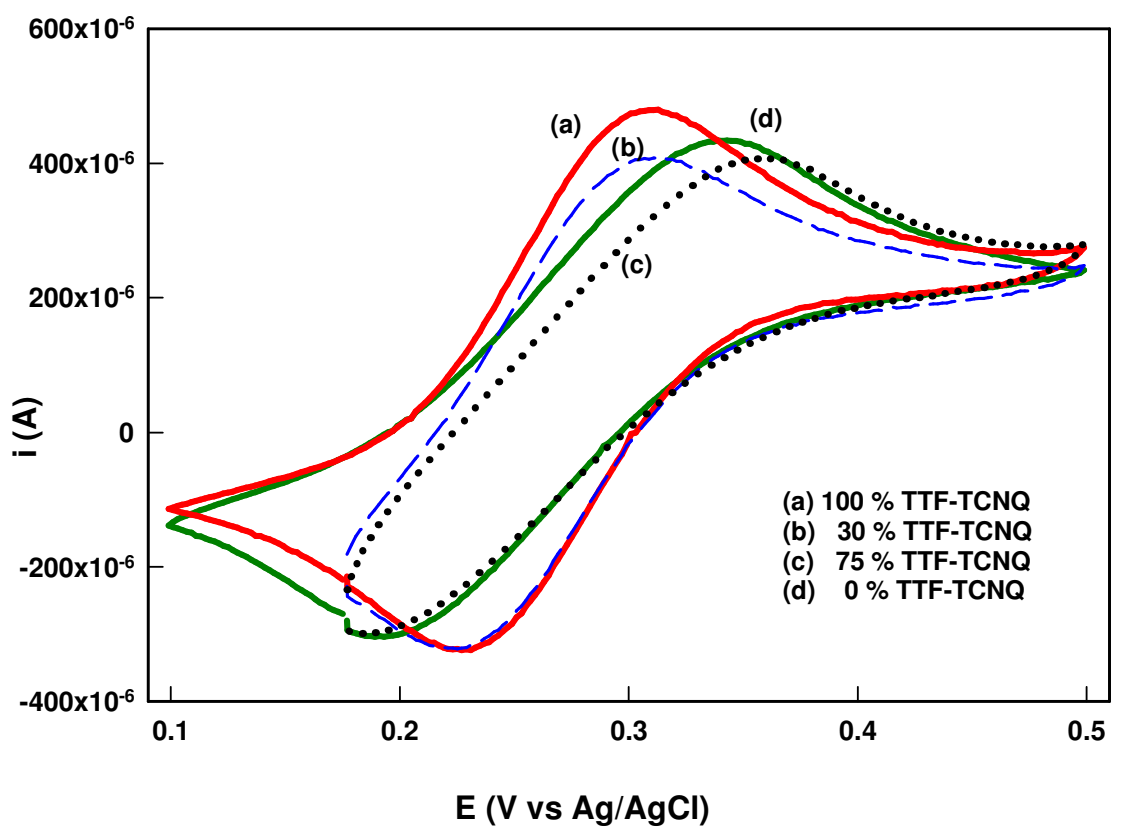

Figura 1. Voltamperogramas cíclicos para $5 \cdot 10^{-2} \mathrm{M}$ de ferrocianuro potásico en $\mathrm{KBr} 1$ $\mathrm{M}$, obtenidos a $v=0.01 \mathrm{~V} \mathrm{~s}^{-1}$ sobre electrodos compósitos de diferentes proporciones TTF-TCNQ/Grafito (\% peso).

La Tabla 1 resume los resultados obtenidos de las curvas de calibración para los distintos electrodos compósitos con una proporción variable de mezcla grafito/TTF-TCNQ y una relación 20/100 (p/p) PVC/mezcla. El rango estudiado de concentraciones de ferrocianuro potásico fue de $2.5 \cdot 10^{-3}$ a $5 \cdot 10^{-2} \mathrm{M}$. Se comprobó que la proporción de TTF-TCNQ en la mezcla no tenía un efecto apreciable sobre la sensibilidad o la precisión. Así, todas las mezclas de grafito/TTF-TCNQ estudiadas proporcionaban electrodos compósitos con similares propiedades electroanalíticas a las del electrodo compósito convencional de PVC/grafito.

Por otro lado, aumentando la proporción de SOC en el electrodo compósito de PVC/(grafito/TTF-TCNQ) se observó que se reducía su estabilidad mecánica. Sin embargo, una mezcla consistente sólo en PVC y TTF-TCNQ (es decir, sin grafito) exhibía una elevada estabilidad y dureza. Estos sorprendentes resultados pueden atribuirse a la interacción entre las partículas del grafito y de la sal TTFTCNQ, la cual dificulta la compactación. Tal disminución de la compactación puede también ser el origen de los elevados coeficientes de variación obtenidos para algunos de los electrodos (ver tabla 1). Por consiguiente, estos resultados 
apuntan a que el electrodo compósito de PVC/TTF-TCNQ puede ser un interesante candidato para su empleo como electrodo de trabajo.

Tabla 1. Resultados analíticos de interés de las curvas de calibración obtenidas para electrodos compósitos con $20 \%$ (p/p) PVC/(grafito + TTF-TCNQ), y proporción variable de grafito/TTF-TCNQ. Datos obtenidos para ferrocianuro potásico en $\mathrm{KBr} 1 \mathrm{M}$, usando una velocidad de barrido de $0.01 \mathrm{Vs}^{-1}$.

\begin{tabular}{ccccccc}
\hline $\begin{array}{c}\text { Grafito/TTF- } \\
\text { TCNQ }(\mathbf{p} / \mathbf{p})\end{array}$ & $\begin{array}{c}\text { Pendiente } \\
(\boldsymbol{\mu} \mathbf{A} / \mathbf{M})\end{array}$ & $\begin{array}{c}\text { Ordenada } \\
(\boldsymbol{\mu A})\end{array}$ & $\mathbf{L O D}^{\mathbf{a}}$ & $\mathbf{S y} / \mathbf{x}^{\mathbf{b}}$ & $\mathbf{R}^{\mathbf{2}}$ & $\mathbf{\mathbf { \% C V } ^ { \mathbf { c } }}$ \\
\hline $100 / 0$ & $8156.38 \pm 150$ & $1.05 \pm 3.9$ & $2.1 \cdot 10^{-3}$ & 5.84 & 0.999 & 1.73 \\
$90 / 10$ & $7338.84 \pm 200$ & $-0.932 \pm 5.2$ & $3.2 \cdot 10^{-3}$ & 7.8 & 0.998 & 5.53 \\
$70 / 30$ & $9144 \pm 148$ & $-9.27 \pm 3.8$ & $1.9 \cdot 10^{-3}$ & 5.7 & 0.999 & 4.27 \\
$50 / 50$ & $8145.34 \pm 75$ & $3.21 \pm 2$ & $1.1 \cdot 10^{-3}$ & 2.91 & 0.999 & 1.75 \\
$35 / 75$ & $7963.21 \pm 172$ & $4.13 \pm 4.4$ & $2.5 \cdot 10^{-3}$ & 6.67 & 0.998 & 9.41 \\
$0 / 100$ & $7895.91 \pm 288$ & $4.95 \pm 7.4$ & $4.2 \cdot 10^{-3}$ & 11.18 & 0.996 & 3.78
\end{tabular}

${ }^{a}$ Concentración, M. ${ }^{b}$ Desviación estándar de residuales. ${ }^{c}$ Coeficientes de variación para 5 medidas.

Tabla 2. Resultados analíticos de interés de las curvas de calibración obtenidas para electrodos compósitos de PVC/TTF-TCNQ, con proporción variable de PVC. Datos obtenidos para ferrocianuro potásico en $\mathrm{KBr} 1 \mathrm{M}$, usando una velocidad de barrido de $0.01 \mathrm{~V} \mathrm{~s}^{-1}$.

\begin{tabular}{ccccccc}
\hline $\begin{array}{c}\text { PVC/TTF- } \\
\text { TCNQ } \\
(\mathbf{g} / \mathbf{1 0 0 g})\end{array}$ & $\begin{array}{c}\text { Pendiente } \\
(\boldsymbol{\mu A} / \mathbf{M})\end{array}$ & $\begin{array}{c}\text { Ordenada } \\
(\boldsymbol{\mu A})\end{array}$ & $\mathbf{L O D}^{\mathbf{a}}$ & $\mathbf{S y} / \mathbf{x}$ & $\mathbf{R}^{\mathbf{b}}$ & $\mathbf{\% C V}^{\mathbf{c}}$ \\
\hline 20 & $7895.91 \pm 288$ & $4.95 \pm 7.4$ & $4.2 \cdot 10^{-3}$ & 11.18 & 0.996 & 3.78 \\
10 & $8392.17 \pm 145$ & $1.20 \pm 3.7$ & $2.0 \cdot 10^{-3}$ & 5.63 & 0.999 & 1.69 \\
5 & $8156.95 \pm 120$ & $-5.56 \pm 3$ & $1.7 \cdot 10^{-3}$ & 4.63 & 0.999 & 4.25 \\
${ }^{\mathrm{a}}$ Concentración, M. ${ }^{\mathrm{b}}$ Desviación estándar de residuales. ${ }^{\mathrm{c}}$ Coeficiente de variación para 5 medidas.
\end{tabular}

Con objeto de mejorar las propiedades del electrodo PVC/TTF-TCNQ, se examinó también la influencia de la proporción del PVC en la mezcla. Aunque aumentando la proporción de PVC se facilitaba la compactación de la mezcla, esto también incrementaba la resistencia eléctrica, en el sentido de distorsionar el potencial de la señal electroanalítica. Así, proporciones de PVC por encima de 20/100 (p/p) proporcionan pobres resultados. Por el contrario, disminuyendo sustancialmente la proporción de PVC se mejora el comportamiento analítico del electrodo compósito, en términos de sensibilidad y de límites de detección (ver Tabla 2). No obstante, una reducción del contenido de PVC por debajo de 5/100 $(\mathrm{p} / \mathrm{p})$ conduce a elevados coeficientes de variación, debido a una inadecuada compactación del material. Por tanto, la proporción de PVC 10/100 (p/p) en el electrodo compósito se presenta como solución de compromiso. 
Influencia de la composición del electrodo en el comportamiento electroquímico Se examinó el efecto de la composición del electrodo compósito sobre su reactividad electroquímica. La Fig. 2 muestra la variación en la separación de los potenciales de pico voltamétricos $\left(\Delta E_{p}=E_{p}{ }^{a}-E_{p}{ }^{c}\right)$, y la corriente de pico de oxidación $\left(i_{p, o x}\right)$ como una función de la relación grafito/TTF-TCNQ $(\mathrm{p} / \mathrm{p})$ en el electrodo compósito, para una disolución de ferrocianuro potásico de concentración de $1 \mathrm{mM}$, bajo idénticas condiciones experimentales. Como puede verse, la mayor reactividad electroquímica (alta $i_{p, o x}$ y bajo valor de $\Delta E_{p}$ ) se obtuvo en ausencia de TTF-TCNQ o grafito, es decir, para un electrodo de PVC/grafito (0\% TTF-TCNQ) o un electrodo PVC/TTF-TCNQ (100\% TTFTCNQ). Por otro lado, los electrodos de grafito/TTF-TCNQ exhiben fluctuaciones que, como se indicó previamente, pueden deberse a interacciones entre partículas de grafito y de TTF-TCNQ.

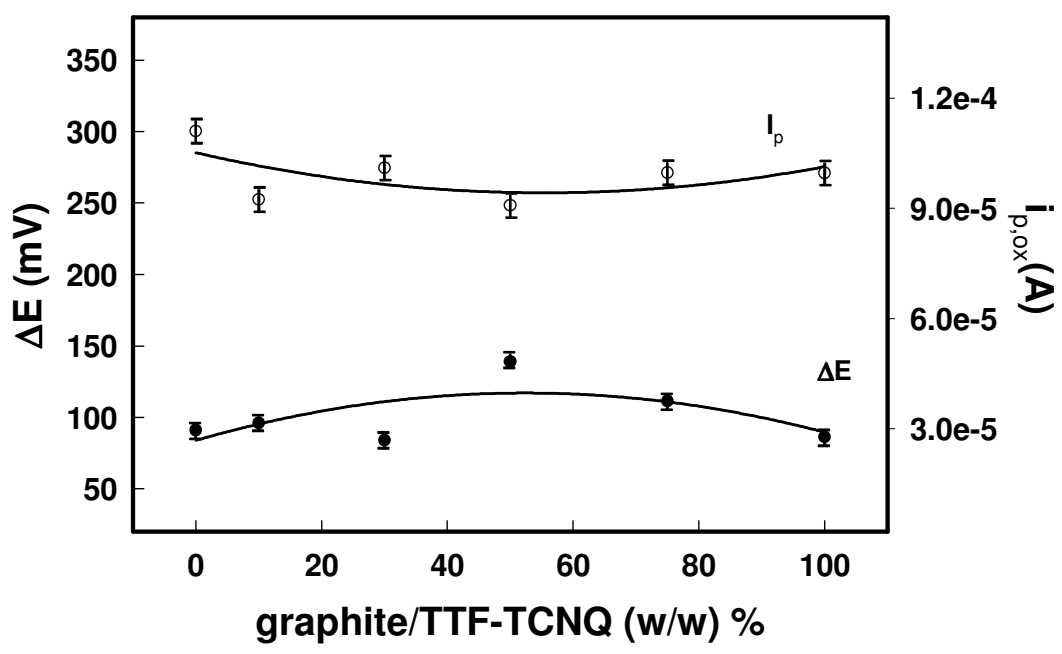

Figura 2. Efecto de la relación grafito/TTF-TCNQ (p/p) \% sobre la intensidad de pico de oxidación, $i_{p, o x}$, y la separación de los potenciales de pico, $\Delta E_{p}$. Datos obtenidos para $1 \mathrm{mM}$ de ferrocianuro potásico en $\mathrm{KBr} 1 \mathrm{M} \mathrm{a} v=0.01 \mathrm{Vs}^{-1}$.

El efecto de la proporción de PVC en el electrodo PVC/TTF-TCNQ puede verse en la Fig. 3. La mayor reactividad electroquímica se obtuvo para la relación 10/100 (p/p) PVC/TTF-TCNQ.

Estos resultados son bastante consistentes con los obtenidos en las gráficas de calibración (ver Tablas 1 y 2). 


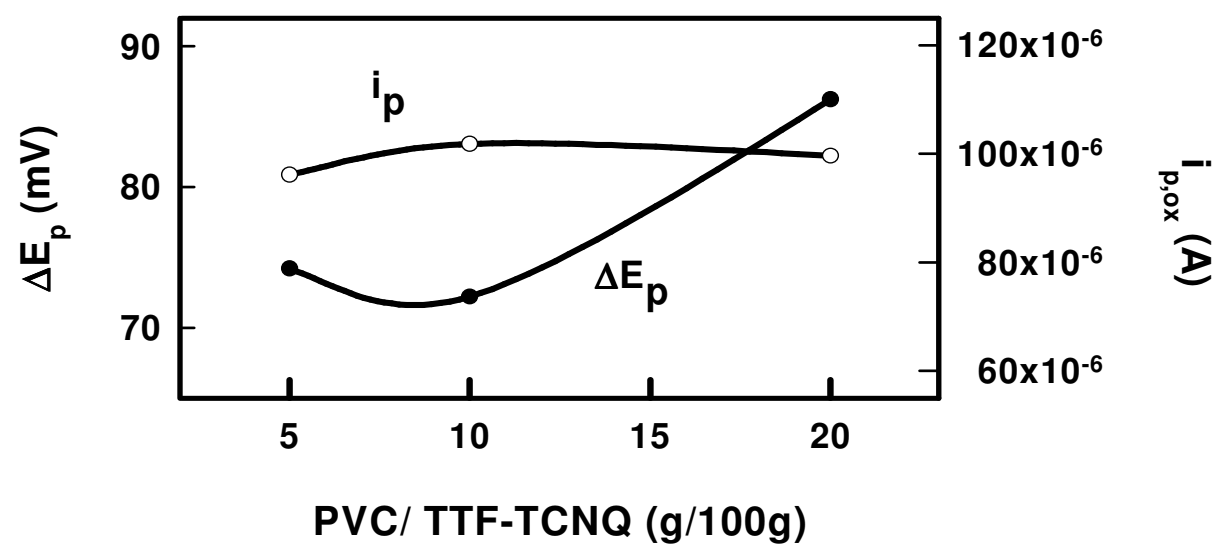

Figura 3. Efecto de la proporción de PVC/TTF-TCNQ (g/100g) sobre la intensidad de pico de oxidación, $i_{p, o x}$, y la separación de los potenciales de pico, $\Delta E_{p}$. Datos obtenidos para $1 \mathrm{mM}$ de ferrocianuro potásico en $\mathrm{KBr} 1 \mathrm{M} . v=0.01 \mathrm{~V} \mathrm{~s}^{-1}$.

La Fig. 1 compara los resultados obtenidos para $5 \cdot 10^{-2} \mathrm{M}$ de ferrocianuro potásico en $\mathrm{KBr} 1 \mathrm{M}$ sobre diversos electrodos compósito. Como puede verse, el electrodo de PVC/TTF-TCNQ (gráfica a) proporciona picos voltamétricos más agudos, y a menor potencial, que el electrodo convencional de $\mathrm{PVC} /$ grafito (gráfica $d$ ). Los electrodos formados por mezclas de grafito/TTF-TCNQ (gráficas b y c) muestran propiedades intermedias, y además, su intervalo de trabajo es algo menor que para los electrodos formados únicamente por grafito o TTFTCNQ.

Teniendo en cuenta estos resultados, el electrodo compósito 10/100 (p/p) PVC/TTF-TCNQ se propone como una interesante alternativa al electrodo convencional de $\mathrm{PVC} /$ grafito, mejorándolo en sensibilidad y precisión. Además, el electrodo propuesto exhibe buena estabilidad mecánica, y su superficie puede regenerarse fácilmente mediante un simple pulido.

La variabilidad entre electrodos se encontró que era inferior al $4 \%$ para electrodos de la misma tanda de preparación, y menos del $7 \%$ para electrodos de diferente lote. También, la variabilidad después de una serie de operaciones de renovación de superficies era inferior al $2 \%$. Por tanto, el electrodo propuesto es, además, altamente reproducible.

Los datos voltamétricos para ferrocianuro potásico se usaron para calcular la constante de velocidad heterogénea $\left(\mathrm{k}_{\mathrm{s}}\right)$, usando el método de Nicholson [17]. Los valores de $k_{s}$ así obtenidos fueron $5.1 \cdot 10^{-4}$ y $5.2 \cdot 10^{-3} \mathrm{~cm} \mathrm{~s}^{-1}$ para el electrodo de PVC/grafito y de PVC/TTF-TCNQ, respectivamente. Estos valores son similares a los encontrados para ferrocianuro sobre electrodos sólidos [18]. El incremento de la constante de velocidad heterogénea obtenido con el electrodo de PVC/TTF-TCNQ es consistente con el desplazamiento negativo observado en el pico de potencial anódico (ver Fig. 1), e indica una menor distancia entre los sitios activos en la superficie del electrodo o, en otras palabras, mayor compactación. 


\section{Aplicaciones. Detección amperométrica de ácido ascórbico en FIA}

La aplicabilidad, como electrodo de trabajo, del electrodo PVC/TTF-TCNQ propuesto se comprobó en la determinación de AA en un sistema FIA. Esta pequeña molécula está presente en muchos sistemas biológicos y en preparaciones multivitamínicas. Además, el AA es usado extensamente como antioxidante alimenticio con objeto de aumentar el tiempo de conservación de algunos productos. Varios electrodos modificados químicamente $[19,20 \mathrm{y}$ referencias en ellas] y electrodos basados en TTF-TCNQ [7-9, 21] han sido propuestos para mejorar su detección electroquímica en célula. Sin embargo, pocos de estos electrodos permiten mejorar la detección de AA en sistemas de inyección en flujo.

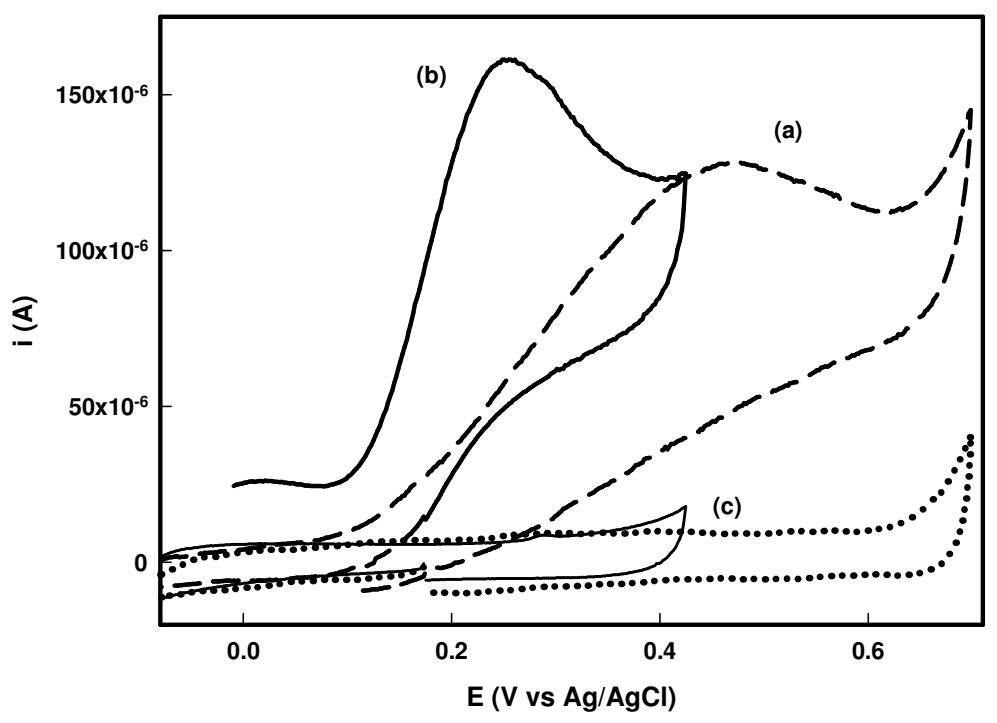

Figura 4. Voltagramas cíclicos para $10 \mathrm{mM}$ AA en $\mathrm{KBr} 0.1 \mathrm{M}$ obtenidos a $v=0.01$ $\mathrm{Vs}^{-1}$ sobre un electrodo de PVC/grafito $(a)$, y sobre un electrodo de PVC/TTF-TCNQ (b). (c) Voltagramas de los correspondientes fondos.

En primer lugar, se efectuaron pruebas voltamperométricas preliminares sobre el ácido ascórbico para identificar el electrolito más apropiado, así como determinar el potencial óptimo a aplicar en términos de sensibilidad. En este sentido, los mejores resultados se obtuvieron empleando $\mathrm{KBr} 0.1 \mathrm{M}$ como electrolito soporte. La Fig. 4 compara los voltagramas para $5 \mathrm{mM}$ AA obtenidos con un electrodo de PVC/grafito (a) y con el electrodo propuesto de PVC/TTF-TCNQ (b). Este último exhibe una mayor intensidad y un menor potencial de pico, debido, posiblemente, al efecto catalítico de la SOC sobre la oxidación del AA.

Teniendo en cuenta los registros anteriores, la mayor respuesta de corriente de pico del electrodo PVC/TTF-TCNQ frente al AA tiene lugar a unos $0.26 \mathrm{~V}$, por lo que este valor de potencial fue seleccionado como el más idóneo en el sistema FIA.

La influencia de las variables hidrodinámicas, tales como la velocidad de flujo y el volumen de inyección, sobre la respuesta del electrodo de PVC/TTF-TCNQ al 
AA también fueron estudiadas. Los mejores resultados, en términos de sensibilidad y reproducibilidad, se obtuvieron a una velocidad de flujo de $1 \mathrm{~mL}$ $\min ^{-1}$ y un volumen de inyección de $140 \mu \mathrm{L}$.

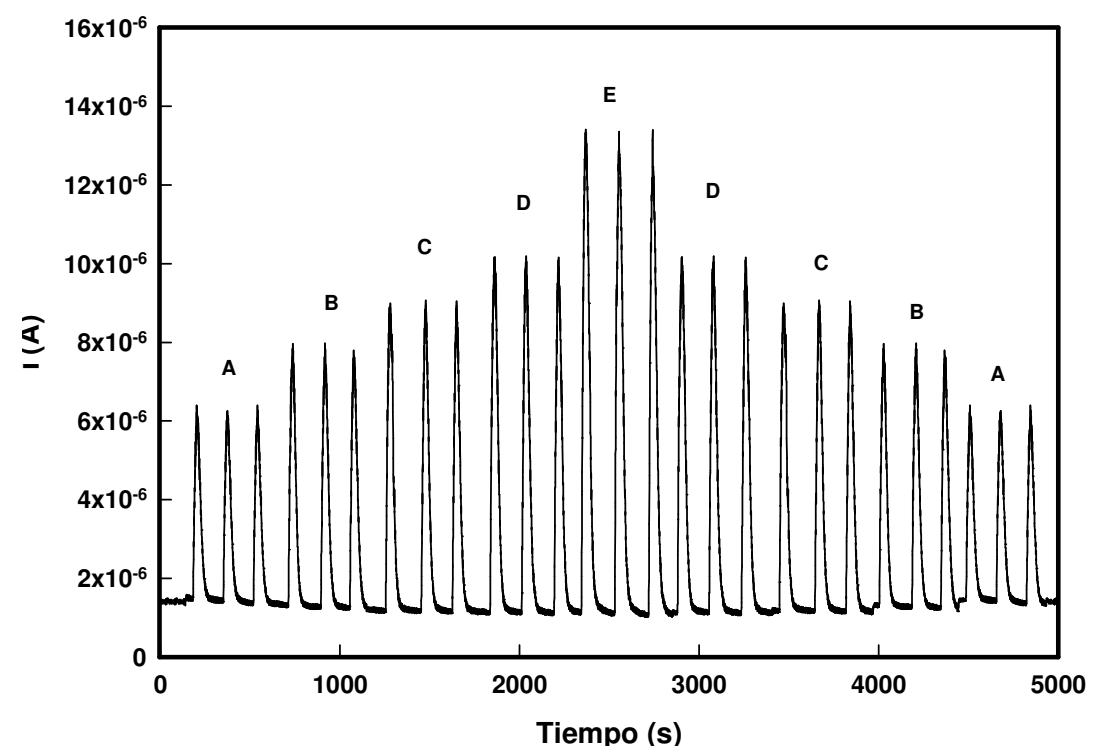

Figura 5. Detección amperométrica en FIA del AA, a concentración variable, sobre un electrodo de PVC/TTF-TCNQ. $A=1.5 \cdot 10^{-4} \mathrm{~mol} \mathrm{~L}^{-1}, B=2 \cdot 10^{-4} \mathrm{~mol} \mathrm{~L}^{-1}, C=2.5 \cdot 10^{-4}$ mol L ${ }^{-1}, D=3 \cdot 10^{-4}$ mol L ${ }^{-1}, E=5 \cdot 10^{-4} \mathrm{~mol} \mathrm{~L}^{-1}$. Portador $\mathrm{KBr} 0.1 \mathrm{M}$. Volumen inyectado $140 \mu \mathrm{L}$. Velocidad de flujo $1 \mathrm{~mL} \mathrm{~min}^{-1}$. Potencial aplicado 0. $26 \mathrm{~V}$.

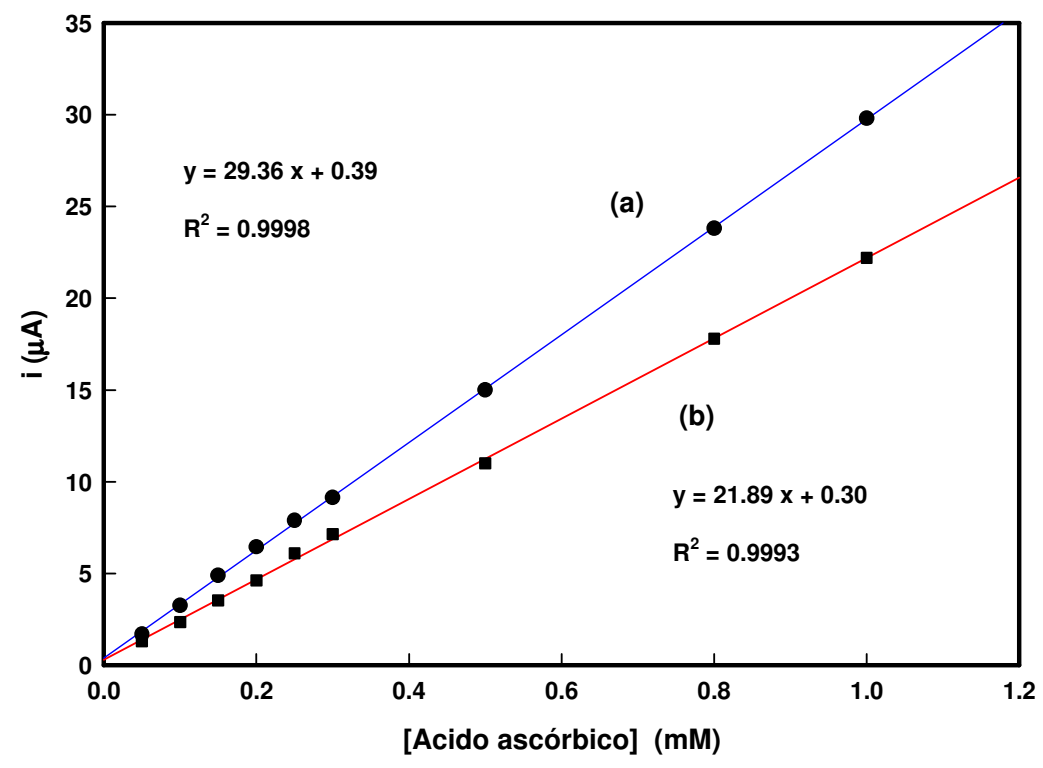

Figura 6. Representaciones de las rectas de calibrado para el ácido ascórbico: (a) sobre el electrodo de PVC/ TTF-TCNQ y (b) sobre PVC/grafito. 
La Fig. 5 muestra las señales amperométricas obtenidas a concentraciones variables de AA en $0.1 \mathrm{M} \mathrm{KBr}$.

La reproducibilidad del sistema fue analizada mediante varias inyecciones consecutivas de una solución $0.5 \mathrm{mM}$ AA. Como resultado, se obtuvo una desviación estándar relativa de $0.46 \%$, la cual indica buena reproducibilidad y, por lo tanto, buena resistencia mecánica del electrodo compósito bajo las condiciones de flujo.

En la Fig. 6 se muestran las rectas de calibrado correspondientes a la determinación amperométrica del AA en $0.1 \mathrm{M} \mathrm{KBr}$ con el electrodo propuesto, y comparándolo con el electrodo de PVC/grafito. Cada estándar usado se midió 5 veces.

Para el electrodo propuesto se obtuvo un límite de detección (definido como la señal del blanco más tres veces su desviación estándar) de $1.51 \cdot 10^{-5} \mathrm{M}$, mientras que para el electrodo de PVC/grafito (el potencial aplicado fue de $0.32 \mathrm{~V}$ ) el límite de detección obtenido fue de $3.18 \cdot 10^{-5} \mathrm{M}$. Para ambos electrodos el rango lineal de determinación fue $5 \cdot 10^{-5}-5 \cdot 10^{-4}$ M. Por tanto, el electrodo propuesto exhibe una alta sensibilidad y una buena respuesta lineal. De hecho, su respuesta analítica supera a la del electrodo convencional de PVC/grafito.

Finalmente, conviene destacar que la metodología propuesta para la preparación de este nuevo electrodo compósito es altamente flexible. En este sentido, pueden desarrollarse mezclas con otras SOC, que permitan construir electrodos eficientes, capaces de ser empleados para detección electroquímica en sistemas FIA.

\section{Conclusiones}

La incorporación de la sal TTF-TCNQ como fase conductora en electrodos compósitos, en forma de pastillas, ha resultado ser un método efectivo para la preparación de electrodos de trabajo alternativo, susceptible de ser usados en sistemas de flujo continuo. El electrodo compósito de PVC/TTF-TCNQ propuesto en este trabajo exhibe muy buenas propiedades electroquímicas, una elevada sensibilidad y también una alta estabilidad mecánica. Además, es fácil y asequible de preparar, posee una elevada estabilidad, y proporciona resultados muy reproducibles, siendo su superficie fácilmente regenerable. Su aplicabilidad en un sistema FIA se ha probado, satisfactoriamente, en la detección amperométrica del AA, consiguiendo mejorar la respuesta del electrodo convencional de PVC/grafito.

\section{Agradecimientos}

Los autores agradecen la financiación del Ministerio de Educación y Ciencia español a través del proyecto CTQ2004-01677, cofinanciado con fondos FEDER. Agradecen también al profesor M. Valcárcel del Departamento de Química Analítica de la Universidad de Córdoba por proporcionar el equipamiento FIA. 


\section{Preparación y Caracterización de un Electrodo Compósito de PVC/TTF-TCNQ. Aplicaciones en un Sistema de Inyección de Flujo}

\section{Resumen}

Se ha preparado un electrodo compósito de cloruro de polivinilo (PVC)/tetratiofulvaleno-tetracianoquinodimetano (TTF-TCNQ) que presenta buenas características físicas y electroanalíticas. El estudio con distintas proporciones de PVC/TTF-TCNQ-grafito ha mostrado que la relación 1/10 (p/p) PVC/TTF-TCNQ es la que mejores resultados ofrece en términos de sensibilidad, coeficientes de variación y de resistencia mecánica. Este electrodo presenta interesantes aplicaciones como electrodo de trabajo. Así, destaca su empleo en sistemas de inyección de flujo, habiéndose aplicado satisfactoriamente a la determinación de ácido ascórbico. También ha mostrado su utilidad como sensor voltamperométrico de iones.

El electrodo propuesto presenta buenas propiedades cinéticas, con una baja corriente de fondo y una señal relativamente reproducible. Además, puede ser preparado de una manera simple, y su superficie es fácilmente regenarable.

Palabras clave: TTF-TCNQ; electrodo compósito; PVC; ácido ascórbico; inyección en flujo.

\section{Referencias}

1. C.D. Jaeger, A.J. Bard, J. Am. Chem. Soc. 101 (1979) 1690.

2. C.D. Jaeger, A.J. Bard, J. Am. Chem. Soc. 102 (1980) 5435.

3. K.B. Scaboo, J.Q. Chambers, Electrochim. Acta 43 (1998) 3257.

4. M. Lamache, H. Menet, A. Moradpour, J. Am. Chem. Soc. 104 (1982) 4520 .

5. P. Calvo-Marzal, K.Y. Chumbimuni-Torres; N.F. Hoehr; G. de-OliveiraNeto, L.T. Kubota, Sens. Actuators B Chem. 100 (2004) 333.

6. W.J. Albery, P.N. Bartlett, D.H. Craston, J. Electroanal. Chem. 194 (1985) 223.

7. K. McKenna, S. Boyette, A. Brajter-Toth, Anal. Chim. Acta 206 (1988) 75.

8. M.S. Freund, A. Brajter-Toth, M.D. Ward, J. Electroanal. Chem. 289 (1990) 127.

9. E. Dabek-Zlotorzynska, K. Ahmad, A. Brajter-Toth, Anal. Chim. Acta 246 (1991) 315.

10. T.R.I. Cataldi, D. Centonze, Anal. Chim. Acta 326 (1996) 107.

11. F. Palmisano, P.G. Zambonin, D. Centonce, M. Quinto, Anal. Chem. 74 (2002) 5913.

12. X. Llopis, A. Merkoci, M. del Valle, S. Alegret, Sens. Actuators B Chem. 107 (2005) 742.

13. M. Luque, E. Luque-Pérez, A. Ríos, M. Valcárcel, Anal. Chim. Acta 410 (2000) 127.

14. B. Palenzuela, R. Rodríguez-Amaro, A. Ríos, M. Valcárcel, Electroanalysis 14 (2002) 1427. 
15. M. Cano, B. Palenzuela, R. Rodríguez-Amaro, Electroanalysis 18 (2006) 1068.

16. F. Albertús, A. Llerena, J. Alpizar, V. Cerdá, M. Luque, A. Ríos, M. Valcarcel, Anal. Chim. Acta 355 (1997) 23.

17. R.S. Nicholson, Anal. Chem. 37 (1965) 1351.

18. J. Wang, Ü.A. Kirgöz, J. Mo, J. Lu, A.N. Kawde, A. Muck, Electrochem. Comm. 3 (2001) 203.

19. J.B. Raoof, R. Ojani, S. Rashid-Nadimi, Electrochim. Acta. 49 (2004) 271.

20. H.R. Zare, F. Memarzadeh, M. Mazloum Ardakani, M. Namazian, S.M. Golabi, Electrochim. Acta 50 (2005) 3495.

21. U. Korell, R.B. Lennox, Anal. Chem. 64 (1992) 147. 